\title{
Local correlations and hole doping in NiO: A dynamical mean-field study
}

\author{
J. Kuneš, ${ }^{1,2, *}$ V. I. Anisimov, ${ }^{3}$ A. V. Lukoyanov, ${ }^{4}$ and D. Vollhardt ${ }^{1}$ \\ ${ }^{1}$ Theoretical Physics III, Center for Electronic Correlations and Magnetism, Institute of Physics, University of Augsburg, \\ Augsburg 86135, Germany \\ ${ }^{2}$ Institute of Physics, Academy of Sciences of the Czech Republic, Cukrovarnická 10, 16253 Praha 6, Czech Republic \\ ${ }^{3}$ Institute of Metal Physics, Russian Academy of Sciences-Ural Division, 620041 Yekaterinburg GSP-170, Russia \\ ${ }^{4}$ Ural State Technical University-UPI, 620002 Yekaterinburg, Russia
}

(Received 23 February 2007; published 24 April 2007)

\begin{abstract}
Using a combination of $a b$ initio band-structure methods and dynamical mean-field theory, we study the single-particle spectrum of the prototypical charge-transfer insulator NiO. Good agreement with photoemission and inverse-photoemission spectra is obtained for both stoichiometric and hole-doped systems. In spite of a large $\mathrm{Ni} d$ spectral weight at the top of the valence band, the doped holes are found to occupy mainly the ligand $p$ orbitals. Moreover, high hole doping leads to a significant reconstruction of the single-particle spectrum accompanied by a filling of the correlation gap.
\end{abstract}

DOI: 10.1103/PhysRevB.75.165115

PACS number(s): 71.27.+a, 71.10.-w, 79.60.-i

\section{INTRODUCTION}

In 1937, at the outset of modern solid-state physics, de Boer and Verwey ${ }^{1}$ drew attention to the surprising properties of materials with incompletely filled $3 d$ bands, such as NiO. This observation prompted Peierls and $\mathrm{Mott}^{2}$ to discuss the interaction between the electrons. Since then, transitionmetal oxides (TMOs) were investigated intensively. This interest further increased when it was discovered that TMOs display an amazing multitude of ordering and electron correlation phenomena, including high-temperature superconductivity, colossal magnetoresistance, and Mott metal-insulator transitions. ${ }^{3}$ In the late $1950 \mathrm{~s}, \mathrm{MnO}$ and $\mathrm{NiO}$ were taken as the textbook examples of antiferromagnets. However, when the importance of local Coulomb correlations in the transition-metal $d$ shell was realized, TMOs were considered candidates for Mott insulators. ${ }^{2}$ In the mid 1980s, Zaanen, Sawatzky, and Allen (ZSA) introduced their classification of TMOs and related compounds into Mott-Hubbard and charge-transfer (CT) systems. ${ }^{4}$ In the early TMOs, the ligand $p$ band is located well below the transition-metal $d$ band and thus plays a minor role in the low-energy dynamics. Such a case, called Mott-Hubbard system in the ZSA scheme, is well described by a multiband Hubbard model. On the other hand, the late TMOs belong to the CT type where the $p$ band is situated between the interaction split $d$ bands. A more general Hamiltonian where the $p$ states are explicitly included is then needed, which can be viewed as a combination of multiband Hubbard and Anderson lattice models. A major impulse for detailed investigations of CT systems, and especially of their hole-doped regime, came with the discovery of hightemperature superconductivity in cuprate perovskites. While the standard three-band Hamiltonian for cuprates ${ }^{5}$ contains only one $d$ orbital per lattice site, the description of cubic transition-metal monoxides, the prominent member of which is $\mathrm{NiO}$, requires the full set of $d$ orbitals. The latter not only are of interest for fundamental research but also play an important role in fields such as geophysics. ${ }^{6}$ Furthermore, recent progress in high-pressure experiments ${ }^{7}$ made the insulator-to-metal transition in some TMOs accessible in the laboratory, providing yet another stimulus for theoretical investigations.

We report a computational study of $\mathrm{NiO}$ combining $a b$ initio band-structure calculations in the local-density approximation (LDA) with the dynamical mean-field theory (DMFT), an approach known as LDA+DMFT. ${ }^{8}$ By treating the local correlations and the $\mathrm{Ni} 3 d-\mathrm{O} 2 p$ hybridization on the same footing, we provide a description of the full valence and conduction band spectra of a CT system with strong hybridization. We will show that a good quantitative agreement with photoemission and inverse-photoemission data can be obtained thereby. This provides a solid foundation for the subsequent investigation of hole doping, a question of broader interest mainly in the context of cuprates. It will be shown that the behavior of the doped holes clearly reveals the CT character of $\mathrm{NiO}$.

$\mathrm{NiO}$ is a type-II antiferromagnet $\left(T_{N}=523 \mathrm{~K}\right)$ with a magnetic moment of almost $2 \mu_{B}$ and a large gap surviving well above $T_{N}$. The standard LDA band theory predicts NiO to be a metal, ${ }^{9}$ or an antiferromagnetic insulator ${ }^{10}$ if spin polarization is allowed. A severe underestimation of the gap and the magnetic moment suggests, however, that the Slater antiferromagnetic state obtained within LDA does not describe the true nature of $\mathrm{NiO}$. On the other hand, exact diagonalization studies on small clusters were quite successful in describing the single- and two-particle spectra, ${ }^{11}$ showing that the local Coulomb interactions are important. This made it clear that an explicit treatment of Coulomb interactions within the $3 d$ shell is needed, and methods such as $\mathrm{LDA}+U,{ }^{12}$ selfinteraction correction, ${ }^{13}$ or GW (Ref. 14) were introduced. The static, orbitally dependent self-energy of LDA $+U$ enforces a separation of the occupied and unoccupied $d$ bands and thus opens a gap comparable to experiment. This, in turn, leads to a significant improvement of the description of static properties such as the local moment or the lattice dynamics. ${ }^{15}$ However, the LDA $+U$ method is limited to an ordered state and does not yield the electronic excitations and the effect of doping correctly.

A systematic inclusion of dynamical correlations was made possible by dynamical mean-field theory. ${ }^{16-18}$ Since its 
introduction, DMFT proved to be a powerful tool for the investigation of electronic systems with strong local correlations. In connection with band-structure methods, the LDA+DMFT scheme ${ }^{8}$ provides access to material specific single-particle spectra as well as more general correlation functions. Applications of LDA+DMFT so far were mostly limited to Mott-Hubbard systems, where the ligand states are integrated out before the correlation problem is solved. Recently, Ren et al. ${ }^{19}$ applied this approach to $\mathrm{NiO}$ and were able to obtain a realistic gap and the near-gap spectra. However, this approach takes into account only $d$ electrons, such that the orbital character of the valence and conduction bands are bound to be the same, the high-frequency incoherent features in the valence band are missing, and the hole doping cannot be described realistically. In this work, we go beyond such limitations by working with the ligand $p$ states explicitly.

\section{COMPUTATIONAL DETAILS}

LDA+DMFT proceeds in two steps: (i) construction of the effective Hamiltonian from converged LDA calculation and (ii) solution of the corresponding DMFT equations. Here, we use the projection onto Wannier functions ${ }^{20}$ to obtain an eight-band $p-d$ Hamiltonian,

$$
\begin{aligned}
H= & \sum_{\mathbf{k}, \sigma}\left(h_{\mathbf{k}, \alpha \beta}^{d d} d_{\mathbf{k} \alpha \sigma}^{\dagger} d_{\mathbf{k} \beta \sigma}+h_{\mathbf{k}, \gamma \delta}^{p p} p_{\mathbf{k} \gamma \sigma}^{\dagger} p_{\mathbf{k} \delta \sigma}+h_{\mathbf{k}, \alpha \gamma}^{d p} d_{\mathbf{k} \alpha \sigma}^{\dagger} p_{\mathbf{k} \gamma \sigma}\right. \\
& \left.+h_{\mathbf{k}, \gamma \alpha}^{p d} p_{\mathbf{k} \gamma \sigma}^{\dagger} d_{\mathbf{k} \alpha \sigma}\right)+\sum_{i, \sigma, \sigma^{\prime}} U_{\alpha \beta}^{\sigma \sigma^{\prime}} n_{i \alpha \sigma}^{d} n_{i \beta \sigma^{\prime}}^{d} .
\end{aligned}
$$

Here, $d_{\mathbf{k} \alpha \sigma}$ and $p_{\mathbf{k} \gamma \sigma}$ are Fourier transforms of $d_{i \alpha \sigma}$ and $p_{i \gamma \sigma}$, which annihilate the $d$ or $p$ electron with orbital and spin indices $\alpha \sigma$ or $\gamma \sigma$ in the $i$ th unit cell, and $n_{i \alpha \sigma}^{d}$ is the corresponding occupation number operator. The elements of $U_{\alpha \beta}^{\sigma \sigma^{\prime}}$ matrix are parametrized by $U$ and $J$. The constrained LDA calculation yields $U=8 \mathrm{eV}$ and $J=1 \mathrm{eV} .{ }^{12}$ To account for the Coulomb interaction already present in LDA, we renormalize the $d d$ diagonal elements of the LDA Hamiltonian by the double counting correction,

$$
h_{\mathbf{k}, \alpha \beta}^{d d}=\widetilde{h}_{\mathbf{k}, \alpha \beta}^{d d}(\mathbf{k})-\left(N_{\text {orb }}-1\right) \bar{U} n_{L D A} \delta_{\alpha \beta},
$$

where $n_{L D A}$ is the average LDA occupation per orbital and $N_{\text {orb }}=10$ is the total number of orbitals within the shell.

Next, we iteratively solve the DMFT equations on the Matsubara contour, a key part of which is the auxiliary impurity problem solved by quantum Monte Carlo (QMC) method. ${ }^{22}$ The results reported here were obtained at $T$ $=1160 \mathrm{~K}$. To obtain the single-particle spectral functions, analytic continuation to real frequencies is performed using the maximum entropy method. ${ }^{23}$ Applying QMC to a gapped system requires careful assessment of ergodicity and autocorrelation issues. ${ }^{24}$

\section{RESULTS AND DISCUSSION}

\section{A. Single-particle spectra}

The orbital occupations, shown in Table I, and the local moment of $1.85 \mu_{B}$ obtained from the paramagnetic DMFT
TABLE I. Orbital occupancies and the local moment on the Ni site for different hole dopings.

\begin{tabular}{lcccc}
\hline \hline$n_{h}$ & $n_{e_{g}}$ & $n_{t_{2 g}}$ & $n_{p}$ & $m_{d}$ \\
\hline 0 & 0.547 & 1.000 & 0.969 & 1.85 \\
0.6 & 0.531 & 0.994 & 0.885 & 1.61 \\
1.2 & 0.530 & 0.980 & 0.800 & 1.45 \\
\hline \hline
\end{tabular}

solution correspond to a $d^{8}$ ground state of the $\mathrm{Ni}$ ion with two ferromagnetically coupled holes of $e_{g}$ symmetry. In Fig. 1 , the calculated spectral densities resolved into Ni $3 d$ and $\mathrm{O} 2 p$ contributions are compared to photoemission and inverse-photoemission data. ${ }^{21}$ Using the full $p$ - $d$ Hamiltonian, we are able to cover the entire valence and conduction band spectra. Features corresponding to $4 s$ and $4 p$ bands at 10 and $13 \mathrm{eV}$, respectively, are not included in the theoretical spectrum. As shown by Eastman and Freeouf, ${ }^{25}$ the relative intensity of the $2 p$ contribution increases with decreasing photon energy. Therefore, the $120 \mathrm{eV}$ spectrum is dominated by Ni $3 d$ emission, while at $66 \mathrm{eV}$ photon energy the $\mathrm{O} 2 p$ contribution peaked around $-4 \mathrm{eV}$ is resolved (for a detailed orbital decomposition, see Ref. 25). The theoretical spectrum very well reproduces the experimental features, including the size of the gap, the $d$ character of the conduction band, the broad $d$ peak at $-9 \mathrm{eV}$, the position of the $p$ band, and the strong $d$ contribution at the top of the valence band. While the gap and the Hubbard subbands can be described already with the static theory $(\mathrm{LDA}+U),{ }^{12}$ a dynamical treatment is apparently needed to capture the substantial redistribution of spectral weight between the incoherent $(-9 \mathrm{eV})$ and resonant $(-2 \mathrm{eV})$ features in the $d$ spectrum. For a more detailed analysis, we show in Fig. 2 the spectral density resolved into $e_{g}$ and $t_{2 g}$ representations. In agreement with

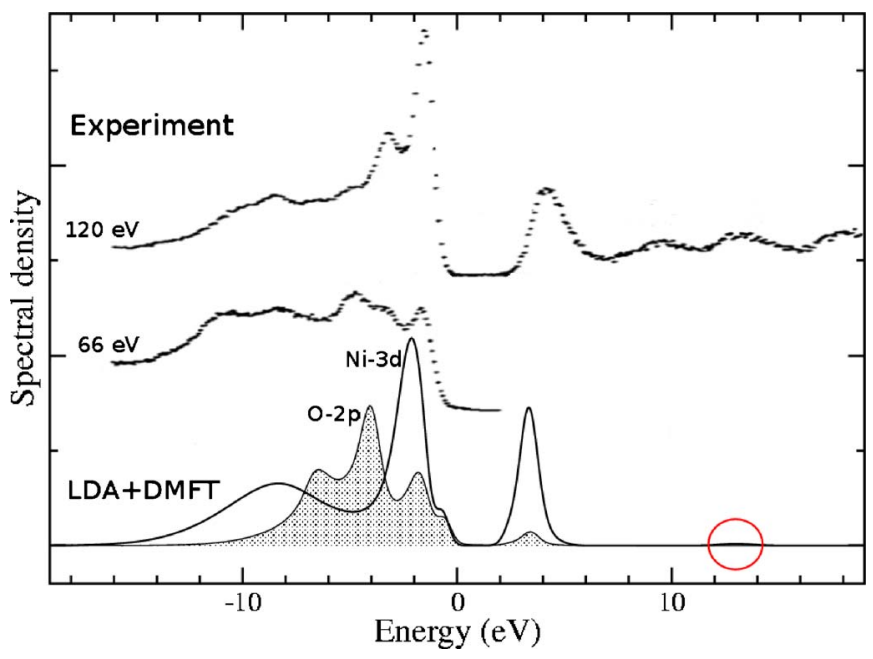

FIG. 1. (Color online) Theoretical Ni $d$ (solid line) and $\mathrm{O} p$ (shaded) resolved spectral densities compared to photoemission and inverse-photoemission data obtained at 120 and $66 \mathrm{eV}$ photon frequencies after Ref. 21. Gaussian broadening of $0.6 \mathrm{eV}$ full width at half maximum corresponding to the experimental resolution was applied to the theoretical curves. The circle marks position of the $d^{10} \underline{L}$ excitation. 


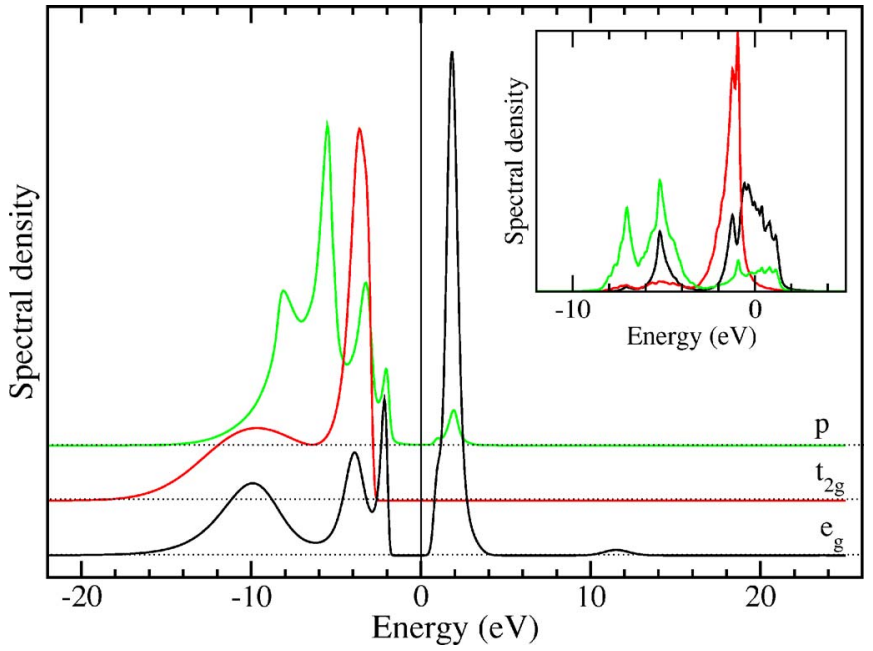

FIG. 2. (Color online) Orbitally resolved O $2 p$ and Ni $3 d$ spectral densities (offset for better resolution). Substantial spectral weight transfer relative to the LDA results (see inset) is observed.

other studies, ${ }^{11,19}$ we find the conduction band to have a pure $e_{g}$ character. The dominant feature of the valence spectrum is a distribution of spectral weight between the broad peak at high frequency and sharp peak(s) at the gap edge. The origin of these structures was discussed by Fujimori et al. ${ }^{11}$ in terms of the eigenstates of a $\mathrm{NiO}_{6}$ cluster. Emitting a $d$ electron from the $d^{8}$ state, the system can end up either directly in a $d^{7}$ final state (broad peak) or in a $d^{8} \underline{L}$ final state (sharp peak), with a ligand hole, due to $p$ - $d$ electron transfer. We use this picture to discuss our DMFT results. A sizable $d^{8} \underline{L}$ resonant peak appears in the filled $t_{2 g}$ band, in spite of only a weak $p-t_{2 g}$ hybridization. This is interpreted as being due to the emission of a $t_{2 g}$ electron followed by an electron transfer from oxygen to the partially filled $e_{g}$ shell. The $e_{g}$ spectrum exhibits even richer structure. In the conduction sector we observe, besides the dominant $d^{9}$ peak, also a tiny highfrequency feature due to the $d^{10} \underline{L}$ final state, an accurate resolution of which is difficult using the maximum entropy method. The resonant $d^{8} \underline{L}$ peak exhibits a pronounced splitting in the $e_{g}$ channel. We ascribe this to the formation of the Zhang-Rice bound state ${ }^{26}$ at the gap edge, which was studied in detail in Ref. 27. Similar effect was also observed on a simpler two band $p$ - $d$ model. ${ }^{28,29}$ In the present work, an accurate resolution of the fine structure of the resonant peak is not possible since the spin-flip terms in the interaction are not included.

\section{B. Hole doping}

Next, we discuss hole doping of NiO. An experimental realization can be found in $\mathrm{Li}_{x} \mathrm{Ni}_{1-x} \mathrm{O}$ studied in the doping range $x=0.02-0.4 .{ }^{30}$ Using the Hamiltonian of the undoped system, the replacement of $x \mathrm{Ni}^{2+}$ ions by $\mathrm{Li}^{1+}$ ions introduces on average $n_{h}=x /(1-x)$ hole per Ni site. As crude as this approximation may be, we believe that the essential physics of $p$ - $d$ weight transfer is captured correctly. In Fig. 3, we show the single-particle spectral densities for $n_{h}=0.6$ corresponding to $x=0.38$. There is no significant difference be-

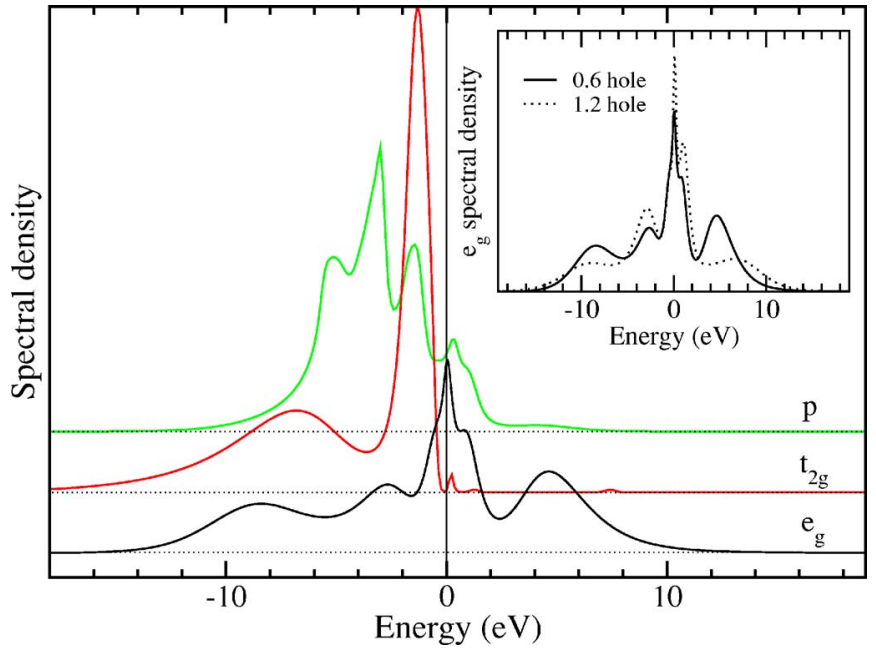

FIG. 3. (Color online) Ni $d$ and $\mathrm{O} p$ resolved spectral densities for a hole concentration $n_{h}=0.6$ (offset for better resolution). The inset shows a comparison of $e_{g}$ spectral densities for hole concentrations $n_{h}=0.6$ and 1.2 .

tween the $t_{2 g}$ spectra in the doped and undoped cases, but the $e_{g}$ spectral function changes significantly. Most notably the Mott gap is filled, while the Hubbard subbands are preserved as distinct features. This is also observed in experiment, as shown in Fig. 4. A quite different behavior was reported in the single-band Mott insulator, ${ }^{31}$ where the gap survives doping while the coherent peak merges with one of the Hubbard subbands. Further hole doping of $\mathrm{NiO}$ leads to a spectral weight transfer from both upper and lower Hubbard subbands to the quasiparticle part of the $e_{g}$ spectrum (see inset of Fig. 3), which can be viewed as an enhancement of the itinerant character of the system reflected also in the decrease of the local moment (see Table I). The $p$ spectral density suggests that much of the doped hole is accommodated on

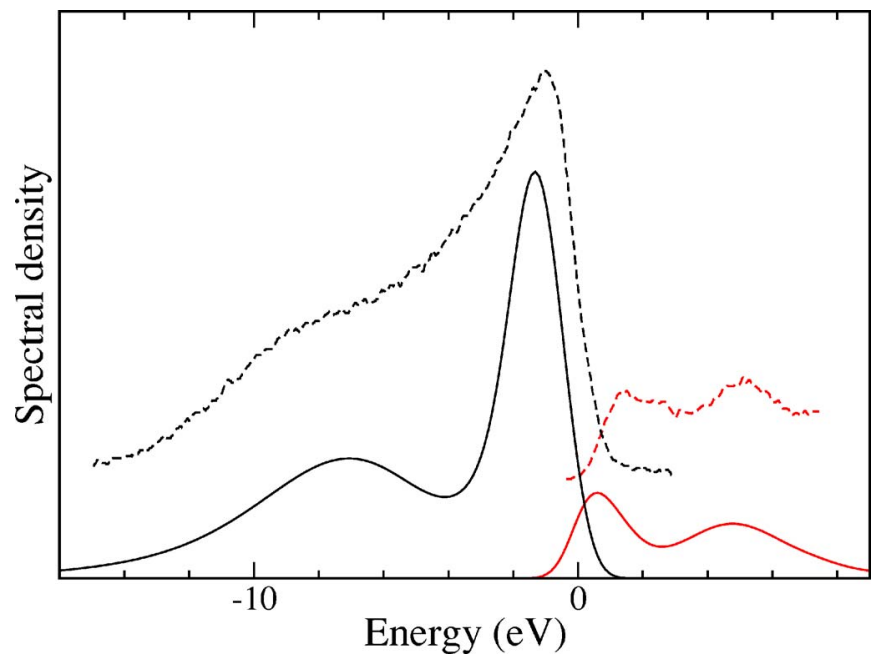

FIG. 4. (Color online) Theoretical Ni $d$ spectral densities for electron addition and electron removal obtained for $n_{h}=0.6$ holedoped $\mathrm{NiO}$ compared to the photoemission and inversephotoemission spectra (Ref. 30) of $\mathrm{Li}_{0.4} \mathrm{Ni}_{0.6} \mathrm{O}$ (the experimental base line is offset for better readability). 
the oxygen sites. That this is indeed the case can be seen from the reduction of the $p$ orbital occupancy shown in Table I. In an uncorrelated system, doping results in a mere shift of the chemical potential and the variation of the orbital occupancy is given by the spectral density at the chemical potential. This reasoning clearly fails in a multiorbital correlated system such as $\mathrm{NiO}$. While the $\mathrm{Ni} d$ spectral density at the top of the valence band in the stoichiometric system (or at the chemical potential in the doped system) is comparable to the $\mathrm{O} p$ spectral density, doped holes almost exclusively reside at the oxygen sites.

\section{CONCLUSIONS}

We used the LDA+DMFT(QMC) approach to compute single-particle spectra of the prototypical CT insulator $\mathrm{NiO}$. By including the ligand $p$ states and the on-site Coulomb interaction within the same framework, we were able to provide a full description of the valence-band spectrum and, in particular, of the distribution of spectral weight between the lower Hubbard band and the resonant peak at the top of the valence band. Good agreement with the available photoemission and inverse-photoemission data was found without the need for adjustable parameters. Importantly, the present method allows us to study the hole doped regime where we find that the doped holes are distributed mainly among the ligand sites. A high hole doping leads to the filling of the correlation gap and a significant transfer of the $d$ spectral weight forms the incoherent part of the spectrum.

\section{ACKNOWLEDGMENTS}

We thank W. E. Pickett and R. T. Scalettar for numerous discussions at the early stage of the code development. J.K. was sponsored by the Alexander von Humboldt Foundation. J.K. and D.V. acknowledge partial support by the SFB 484 of the Deutsche Forschungsgemeinschaft. V.J.A. and A.V.L. were supported by the Russian Foundation for Basic Research under Grants No. RFFI-06-02-81017, No. RFFI-0402-16096, and No. RFFI-03-02-39024 and by the Netherlands Organization for Scientific Research through NWO 047.016.005. A.V.L. acknowledges support from the Dynasty Foundation and International Center.
*Electronic address: jan.kunes@physik.uni-augsburg.de

${ }^{1}$ J. H. de Boer and E. J. W. Verwey, Proc. Phys. Soc. London 49, 59 (1937).

${ }^{2}$ N. F. Mott, Proc. Phys. Soc. London 49, 57 (1937).

${ }^{3}$ For a review, see M. Imada, A. Fujimori, and Y. Tokura, Rev. Mod. Phys. 70, 1039 (1998).

${ }^{4}$ J. Zaanen, G. A. Sawatzky, and J. W. Allen, Phys. Rev. Lett. 55, 418 (1985).

${ }^{5}$ V. J. Emery, Phys. Rev. Lett. 58, 2794 (1987).

${ }^{6}$ R. E. Cohen, I. I. Mazin, and D. G. Isaak, Science 275, 654 (1997).

${ }^{7}$ C. S. Yoo, B. Maddox, J.-H. P. Klepeis, V. Iota, W. Evans, A. McMahan, M. Y. Hu, P. Chow, M. Somayazulu, D. Häusermann et al., Phys. Rev. Lett. 94, 115502 (2005).

${ }^{8}$ K. Held, I. A. Nekrasov, G. Keller, V. Eyert, N. Blümer, A. K. McMahan, R. T. Scalettar, T. Pruschke, V. I. Anisimov, and D. Vollhardt, Phys. Status Solidi B 243, 2599 (2006); G. Kotliar, S. Y. Savrasov, K. Haule, V. S. Oudovenko, O. Parcollet, and C. A. Marianetti, Rev. Mod. Phys. 78, 865 (2006).

${ }^{9}$ L. F. Mattheiss, Phys. Rev. B 5, 290 (1972).

${ }^{10}$ K. Terakura, T. Oguchi, A. R. Williams, and J. Kubler, Phys. Rev. B 30, 4734 (1984).

${ }^{11}$ A. Fujimori, F. Minami, and S. Sugano, Phys. Rev. B 29, 5225 (1984)

${ }^{12}$ V. I. Anisimov, J. Zaanen, and O. K. Andersen, Phys. Rev. B 44, 943 (1991).

${ }^{13}$ A. Svane and O. Gunnarsson, Phys. Rev. Lett. 65, 1148 (1991).

${ }^{14}$ F. Aryasetiawan and O. Gunnarsson, Phys. Rev. Lett. 74, 3221
(1995).

${ }^{15}$ S. Y. Savrasov and G. Kotliar, Phys. Rev. Lett. 90, 056401 (2003).

${ }^{16}$ W. Metzner and D. Vollhardt, Phys. Rev. Lett. 62, 324 (1989).

${ }^{17}$ A. Georges, G. Kotliar, W. Krauth, and M. J. Rozenberg, Rev. Mod. Phys. 68, 13 (1996).

${ }^{18}$ G. Kotliar and D. Vollhardt, Phys. Today 57(3), 53 (2004).

${ }^{19}$ X. Ren, I. Leonov, G. Keller, M. Kollar, I. Nekrasov, and D. Vollhardt, Phys. Rev. B 74, 195114 (2006).

${ }^{20}$ V. I. Anisimov, D. E. Kondakov, A. V. Kozhevnikov, I. A. Nekrasov, Z. V. Pchelkina, J. W. Allen, S.-K. Mo, H.-D. Kim, P. Metcalf, S. Suga et al., Phys. Rev. B 71, 125119 (2005).

${ }^{21}$ G. A. Sawatzky and J. W. Allen, Phys. Rev. Lett. 53, 2339 (1984).

${ }^{22}$ J. E. Hirsch and R. M. Fye, Phys. Rev. Lett. 56, 2521 (1986).

${ }^{23}$ M. Jarrell and J. E. Gubernatis, Phys. Rep. 269, 133 (1996).

${ }^{24}$ J. Kuneš (unpublished).

${ }^{25}$ D. E. Eastman and J. L. Freeouf, Phys. Rev. Lett. 34, 395 (1975).

${ }^{26}$ F. C. Zhang and T. M. Rice, Phys. Rev. B 37, 3759 (1988).

${ }^{27}$ J. Bała, A. M. Oleś, and J. Zaanen, Phys. Rev. Lett. 72, 2600 (1994).

${ }^{28}$ J. Kuneš (unpublished).

${ }^{29}$ M. B. Zölfl, T. Maier, T. Prunschke, and J. Keller, Eur. Phys. J. B 7, 377 (1999).

${ }^{30}$ J. van Elp, H. Eskes, P. Kuiper, and G. A. Sawatzky, Phys. Rev. B 45, 1612 (1992).

${ }^{31}$ T. Pruschke, D. L. Cox, and M. Jarrell, Phys. Rev. B 47, 3553 (1993). 